\title{
Disponibilidade Biológica do Fósforo de Fontes Fosfatadas Determinada por Intermédio da Técnica de Diluição Isotópica. II. Suínos em Crescimento ${ }^{1 *}$
}

\author{
Agustinho Valente de Figueirêdo ${ }^{2}$, Dorinha Miriam S. S. Vitti ${ }^{3}$, João Batista Lopes², \\ Hacy Pinto Barbosa ${ }^{4}$
}

\begin{abstract}
RESUMO - Foram usados 24 leitões machos, castrados, com peso médio inicial de 45,95 kg, para determinar as perdas endógenas fecais, as taxas de absorção verdadeira do fósforo dietético e a disponibilidade biológica do fósforo dos fosfatos. Foi usado o delineamento experimental em blocos casualizados, com seis tratamentos e quatro repetições, e um animal por unidade experimental (gaiola). As rações eram à base de milho e farelo de soja, e o P suplementado através dos fosfatos: bicálcico (BIC); Tapira (TAP); Patos de Minas (FPM); monoamônio (MAP) e supertriplo (SPT) e a dieta controle sem P suplementar (CONT). No $7^{\circ}$ dia do experimento, os animais receberam injeção com 7,4 MBq de ${ }^{32} \mathrm{P}$, como fosfato de sódio. Amostras de sangue, fezes e urina, foram coletadas por sete dias. Concluiu-se que a disponibilidade biológica foi de 71,94; 76,67; 69,49; 64,92 e 66,35\% para os fosfatos BIC, TAP, FPM, MAP E SPT, respectivamente. As variáveis estudadas apresentaram respostas similares entre as diferentes fontes de fósforo suplementar.
\end{abstract}

Palavras-chave: fósforo, diluição isotópica, endógeno, metabolismo, radiofósforo, suínos

\section{Biological Availability of Phosphorus from Various Sources of Phosphate Based on Isotopic Dilution Technique. II. Growing Swine}

\begin{abstract}
Twenty four male castrated pigs, $45.95 \mathrm{~kg}$ of weight live, were used to determine the minimum fecal endogenous phosphorus losses, the rate of true absorption feed and the biological availability of various phosphate sources. A complete randomized block design, with six treatments and four replicates with one animal per experimental unit, was used. The diets were based on corn and soybean meal. The phosphorus supplement of the following sources were used: dicalcium (BIC); Tapira (TAP); Patos Minas (FPM); monoammonium (MAP) e triple superphosphate (SPT) and the diet control without P supplementation (CONT). At the $7^{\text {th }}$ experimental day, the animals were intravenously injected with 7.4 MBq of ${ }^{32} \mathrm{P}$ as sodium phosphate. Samples of blood, feces and urine were collected for seven day. It was concluded that the biological availability was 71.94, 76.67, 69.49, 64.92 and 66.35\% for phosphates BIC, TAP, FPM, MAP and SPT, respectively. The variables assessment showed similar responses to various sources of phosphate.
\end{abstract}

Key Words: endogenous, isotopic dilution, metabolism, phosphorus, radiophosphorus, swine

\section{Introdução}

O fósforo, normalmente, é adicionado às rações dos monogástricos, representando de 1,0 a $1,5 \%$ das despesas totais com alimentação dos suínos. Portanto, o conhecimento do seu valor biológico nos ingredientes é de fundamental importância para a formulação de dietas que possam fornecer quantidades adequadas deste nutriente.

Existem vários fatores que interferem na absorção do fósforo e, a quantidade absorvida pelo animal depende do nível de $\mathrm{P}$ na dieta, da fonte desse mineral, da relação $\mathrm{Ca}: \mathrm{P}$ na dieta, do $\mathrm{pH}$ intestinal e outros elementos minerais que são antagônicos à absorção e utilização de fósforo ( DeLUCA, 1979). A disponibilidade biológica do fósforo é medida pela condição do elemento mineral em suportar algum processo fisiológico, e é definida como sendo a proporção da quantidade de um elemento ingerido de uma dada fonte ou dieta, o qual é absorvido e utilizado pelo animal para atender seu requerimento líquido (PELLER, 1972; PARTRIDGE, 1980). Entre as técnicas utilizadas para se conhecer a disponibilidade biológica mineral, a diluição isotópica é a única que torna possível a determinação das perdas endógenas do elemento, e essas perdas mínimas são importantes para se avaliar as exigências nutricionais dos animais (VITTI, 1989).

Algumas pesquisas têm sido feitas, no Brasil, com

\footnotetext{
${ }^{1}$ Projeto financiado pela FAPESP

${ }_{2}$ Professor do Departamento de Zootecnia/UFPI - 64.049-550 -Teresina, PI. E.mail: lopesjb@uol.com.br

3 Pesquisadora do CENA/USP, CX. Postal 96 - Piracicaba - SP. E.mail: dovitti@cena.usp.br

${ }^{4}$ Pesquisador do Instituto de Zootecnia - Nova Odessa - SP.

* Parte da tese de Doutorado do primeiro autor.
} 
respeito à determinação dos valores de disponibilidade biológica do fósforo dos fosfatos, apresentam, contudo, resultados contraditórios, o que tem dificultado o uso mais amplo dos mesmos em rações de suínos e aves. Isto se deve a diferentes métodos e variáveis de resposta animal usados para determinar os índices de utilização de fósforo pelos animais. Dentre as variáveis de resposta animal destacam-se o ganho em peso e a conversão alimentar (boa sensibilidade); resistência à quebra do osso (alta sensibilidade); fósforo e \% de cinza no osso (alta sensibilidade em leitões jovens e em crescimento); fósforo no soro (baixa sensibilidade) e fosfatase alcalina no soro (boa sensibilidade); outros critérios, também, são usados, como o balanço metabólico, o coeficiente de digestibilidade aparente ou verdadeira e a técnica de diluição isotópica com ${ }^{32} \mathrm{P}$ (CRENSHAW et al. 1981a; MAXSON e MAHAN, 1983; LOPES, 1998).

Usando a técnica com radiofósforo, FERNÁNDEZ (1995a) verificou que a absorção de fósforo foi regulada no intestino de maneira similar à do cálcio, sendo que o balanço de fósforo foi, também, regulado pela ação renal. Através da técnica com ${ }^{32} \mathrm{P}$ e suínos em crescimento, BELLAVER et al ( 1983 ) verificaram que a disponibilidade biológica (DB) do fósforo alimentar foi de 45,86 e $48,22 \%$, para as dietas com e sem $\mathrm{P}$ suplementar, respectivamente. Ainda, de acordo com BELLAVER et al (1984) não houve diferenças significativas para as taxas de absorção verdadeira de 55,$83 ; 60,68 ; 60,39 ; 61,14$ e $66,26 \%$, dos fosfatos Goiás, Patos de Minas, Tapira, da farinha de osso e da dieta testemunha (sem $\mathrm{P}$ suplementar), respectivamente, e nem para os valores de DB do P suplementar dessas fontes, os quais foram de 37,56; 44,30; 47,82; e $46,34 \%$, respectivamente.

O presente trabalho foi desenvolvido com o objetivo de determinar as perdas endógenas fecais mínimas, as taxas de absorção real do fósforo alimentar e a disponibilidade biológica do fósforo dos fosfatos.

\section{Material e Métodos}

O experimento foi realizado nas instalações do Centro de Energia Nuclear na Agricultura (CENA) da USP, utilizando-se 24 suínos machos, castrados, com peso vivo médio de 45,95 kg. Os animais foram mantidos em gaiolas individuais de metabolismo por 14 dias, sendo sete de adaptação e sete de coletas de sangue, fezes e urina. O delineamento experimental foi o de blocos casualizados com seis tratamentos e quatro repetições, e um animal por unidade experimental.

As rações experimentais consistiram de milho, farelo de soja e de trigo, suplementadas com premixes mineral e vitamínico, de forma a atender às exigências dos suínos de acordo com ROSTAGNO et al (1987), exceto a dieta controle, que não recebeu fósforo suplementar (CONT). Os demais tratamentos constaram da suplementação de fósforo através dos fosfatos: bicálcico (BIC); Tapira (TAP); Patos de Minas (FPM); monoamônio (MAP) e supertriplo (SPT). A composição centesimal das rações experimentais e a composição química das fontes fosfatadas, encontram-se nas Tabelas 1 e 2, respectivamente.

As dietas antes de serem fornecidas eram umedecidas com água (1:1 água/ração) e em duas refeições diárias, levando-se em consideração o menor consumo observado na fase pré-experimental, entre os animais de cada bloco. A água foi fornecida à vontade. No sétimo dia do experimento, foram injetados, em cada animal, via endovenosa, 7,4 MBq de ${ }^{32} \mathrm{P}$, na forma de fosfato de sódio $\left(\mathrm{Na}_{2} \mathrm{HPO}_{4}\right)$, livre de carregador. As coletas de sangue (veia cava), fezes e urina foram feitas a cada 24 horas, durante os sete dias experimentais. A detecção da radioatividade nas amostras de fezes, urina e plasma, foi realizada em espectrômetro de cintilação líquida por efeito Cerenkov (IAEA, 1979). As análises bromatológicas dos ingredientes e das dietas seguiram as recomendações da ASSOCIATION OF OFFICIAL ANALYTICAL CHEMISTS - AOAC (1980).

O conteúdo de fósforo inorgânico no plasma, nos fosfatos e na urina, foi determinado por colorimetria, segundo FISKE e SUBBARROW (1925), e nas dietas e nas fezes, pelo método vanadato-molibidato (SARRUGE e HAAG, 1974).

Os cálculos do percentual da atividade do ${ }^{32} \mathrm{P}$ injetada no plasma, nas fezes e as atividades específicas (AE) no plasma e nas fezes, foram feitos de acordo com LOFGREEN (1960), e o valor do fósforo endógeno fecal com base em COMAR et al. (1953) e VITTI (1989). A contagem da atividade do ${ }^{32} \mathrm{P}$ foi realizada por efeito Cerenkov (NASCIMENTO FILHO e LOBÃO, 1977; IAEA, 1979)

A atividade injetada $\left(\mathrm{AI}{ }^{32} \mathrm{P}\right)=[$ (contagem por minuto de $1 \mathrm{~mL}$ de plasma ou $1 \mathrm{~g}$ de fezes/contagem por minuto de uma solução radioativa padrão) x 100 ].

$$
\mathrm{AE}={ }^{32} \mathrm{P} / \mathrm{P} \text { total; }
$$

$\% \mathrm{P}$ endógeno fezes $=\left(\right.$ atividade específica do ${ }^{32} \mathrm{P}$ das fezes / atividade específica do ${ }^{32} \mathrm{P}$ do plasma) x 100 ; 
Tabela 1 - Composição centesimal das dietas experimentais - suínos em crescimento Table 1 - Centesimal composition of the diets - growing swines

\begin{tabular}{|c|c|c|c|c|c|c|}
\hline \multirow[b]{2}{*}{$\begin{array}{l}\text { Ingredientes - } \mathrm{kg} \\
\text { Ingredients }\end{array}$} & \multicolumn{6}{|c|}{$\begin{array}{c}\text { Tratamentos } \\
\text { Treatments }\end{array}$} \\
\hline & BIC & TAP & FPM & MAP & SPT & CONT \\
\hline $\begin{array}{l}\text { Milho } \\
\text { Corn }\end{array}$ & 79,70 & 79,45 & 79,60 & 79,65 & 79,36 & 79,80 \\
\hline $\begin{array}{l}\text { Farelo de soja } \\
\text { Soybean meal }\end{array}$ & 12,10 & 12,00 & 12,10 & 12,40 & 12,30 & 11,30 \\
\hline $\begin{array}{l}\text { Farelo de trigo } \\
\text { Wheat meal }\end{array}$ & 5,36 & 6,00 & 5,20 & 4,50 & 5,20 & 6,30 \\
\hline $\begin{array}{l}\text { Calcário } \\
\text { Limestone }\end{array}$ & 1,02 & 0,30 & 0,40 & 1,70 & 1,30 & 1,70 \\
\hline $\begin{array}{l}\text { Fosf. bicálcico } \\
\text { Dicalcium phosphate }\end{array}$ & 0,92 & - & - & - & - & - \\
\hline $\begin{array}{l}\text { Fosf. Tapira } \\
\text { Tapira }\end{array}$ & - & 1,35 & - & - & - & - \\
\hline $\begin{array}{l}\text { Fosf. Patos Minas } \\
\text { Patos Minas }\end{array}$ & - & - & 1,80 & - & - & - \\
\hline $\begin{array}{l}\text { Fosf.monoamônio } \\
\text { Momoammonium }\end{array}$ & - & - & - & 0,85 & - & - \\
\hline $\begin{array}{l}\text { Fosf. supertriplo } \\
\text { Triple superphosphate }\end{array}$ & - & - & - & - & 0,94 & - \\
\hline $\begin{array}{l}\mathrm{Sal}-\mathrm{NaCl} \\
\text { Salt }\end{array}$ & 0,40 & 0,40 & 0,40 & 0,40 & 0,40 & 0,40 \\
\hline $\begin{array}{l}\text { Suplemento mineral }{ }^{1} \\
\text { Mineral suplement }\end{array}$ & 0,10 & 0,10 & 0,10 & 0,10 & 0,10 & 0,10 \\
\hline $\begin{array}{l}\text { Suplemento vitamínico }{ }^{2} \\
\text { Vitamin suplement }\end{array}$ & 0,40 & 0,40 & 0,40 & 0,40 & 0,40 & 0,40 \\
\hline $\begin{array}{l}\text { Proteína bruta }(\%) \\
\text { Crude protein }\end{array}$ & 14,03 & 14,08 & 14,00 & 14,00 & 14,06 & 14,04 \\
\hline $\begin{array}{l}\text { Energia digestível } \\
\text { Digestible energy }\end{array}$ & 3305 & 3307 & 3299 & 3296 & 3297 & 3301 \\
\hline $\begin{array}{l}\mathrm{Ca}(\%) \\
\mathrm{P} \text { total }(\%)\end{array}$ & 0,61 & 0,61 & 0,62 & 0,62 & 0,61 & 0,61 \\
\hline $\begin{array}{l}\text { P total }(\%) \\
\text { Total P }\end{array}$ & 0,47 & 0,47 & 0,47 & 0,47 & 0,47 & 0,31 \\
\hline
\end{tabular}

${ }^{1}$ Suplemento mineral forneceu por kg de dieta (Mineral suplement to provided per kilogram of diet): Fe 80 mg; Cu 12 mg; Mn 70 mg; Zn 100 mg; I 1 mg; Se $0,12 \mathrm{mg}$.

2 Suplemento vitamínico forneceu por $\mathrm{kg}$ de dieta (Vitamin supplement to provided per kilogram of diet): Vit A $7000 \mathrm{UI}$; Vit $\mathrm{D}_{3} 1200 \mathrm{UI}$; Vit. E $12 \mathrm{mg}$; Vit $\mathrm{K}_{3} 1,6 \mathrm{mg}$; Vit B $1 \mathrm{mg}$; Vit B 3 mg; Vit B $1 \mathrm{mg}$; Vit B $1212 \mathrm{mg}$; Niacina (Niacin) $20 \mathrm{mg}$; Ác. Pantotênico (Panthothenic ac.) $12 \mathrm{mg}$; Colina (Cholin) $150 \mathrm{mg}$; Promotor crescimento (Growth promoter) $48 \mathrm{mg}$; Antioxidante (Antioxidant) $15 \mathrm{mg}$.

$\mathrm{P}$ endógeno total nas fezes $=\%$ do $\mathrm{P}$ endógeno nas fezes x P total excretado nas fezes.

As taxas de absorção real do fósforo foram determinadas pela relação entre a absorção real do fósforo e o $\mathrm{P}$ consumido, e a disponibilidade biológica do fósforo dos fosfatos foi encontrada através de fracionamento do $\mathrm{P}$ consumido e o absorvido real entre a dieta controle e as dietas contendo as fontes fosfatadas (FIGUEIRÊDO, 1998).

\section{Resultados e Discussão}

Pode-se observar que o consumo de ração não foi influenciado pelas fontes fosfatadas $(\mathrm{P}>0,05)$, enquanto os leitões da dieta controle (CONT) consumiram menos ração $(p<0,01)$ do que aqueles dos demais tratamentos (Tabela 3). Por outro lado, BELLAVER et al. (1983) não identificaram diferenças no consumo de alimento entre as rações com e sem $P$ suplementar.

$\mathrm{O}$ consumo de fósforo, entre as dietas que tiveram fósforo suplementar, não diferiu entre si $(\mathrm{P}>0,05)$. Contudo, os leitões da dieta controle (CONT), consumiram menos fósforo $(\mathrm{P}<0,01)$ do que os dos tratamentos com fosfatos. A média de fósforo total consumido entre as rações suplementadas com as fontes fosfatadas foi de 7,0 g/dia, estando este valor dentro dos limites das exigências estabelecidas pelo NRC 
Tabela 2 - Composição química das fontes de fósforo e cálcio ${ }^{1}$

Table 2 - Chemical composition of the phosphorus and calcium sources

\begin{tabular}{lcccccc}
\hline $\begin{array}{l}\text { Fontes } \\
\text { Sources }\end{array}$ & $\mathrm{Ca}(\%)$ & $\mathrm{Pt}(\%)$ & $\mathrm{F}(\%)$ & $\mathrm{P}: \mathrm{F}$ & $\mathrm{Ca}: \mathrm{P}$ & $\mathrm{P}_{2} 0_{5}(\%) *$ \\
\hline $\begin{array}{l}\text { Fosf. bicálcico } \\
\begin{array}{l}\text { Dicalcium } \\
\text { F. Tapira }\end{array}\end{array}$ & 23,1 & 20,0 & 0,09 & $2,27: 1$ & $1,16: 1$ & 42,80 \\
$\begin{array}{l}\text { Tapira } \\
\text { Fosf. Patos de Minas }\end{array}$ & 33,0 & 13,4 & 0,97 & $13,9: 1$ & $2,46: 1$ & 36,47 \\
$\begin{array}{l}\text { Patos de Minas } \\
\text { Fosf. monoamônio }\end{array}$ & 23,3 & 10,4 & 1,73 & $6,0: 1$ & $2,24: 1$ & 23,52 \\
$\begin{array}{l}\text { Momoammonium } \\
\begin{array}{l}\text { Fosf. supertriplo } \\
\text { Triple superphosphate }\end{array}\end{array}$ & 0,5 & 22,2 & 0,51 & $43,5: 1$ & $0,03: 1$ & 56,46 \\
$\begin{array}{l}\text { Calcário } \\
\text { Limestone }\end{array}$ & 12,6 & 20,3 & 0,39 & $52,1: 1$ & $0,62: 1$ & 47,62 \\
\hline
\end{tabular}

${ }^{1}$ Análise foi realizada no laboratório de Nutrição Animal do Centro de Energia Nuclear na Agricultura.

CENA / USP. Analisis was carried out at Animal Nutrition Lab of CENA/USP.

2 As fontes de fósforo foram doadas pela Petrofértil, instalada em Uberaba - MG - Brasil.

* Dados obtidos de Tabelas (Data obtained from Tables).

(1988), de 4,0 g P/kg de ração, para a fase de terminação. A ingestão de fósforo está associada ao seu nível dietético na ração, conforme foi constatado em várias pesquisas (CUPÁK et al. 1972; TONROY et al. 1973; FERNÁNDEZ 1995a; LOPES 1998).

De qualquer modo, o consumo de apenas $2,96 \mathrm{~g}$ $\mathrm{P} /$ dia, obtido para a dieta controle, pode ser considerado baixo para essa categoria de peso dos leitões, pois BELLAVER et al. (1983) e BELLAVER et al. (1984), obtiveram consumo, em dietas semelhantes, de 3,10 e 3,97 g P/dia, para leitões de 21,16 e 29,53 kg de peso, respectivamente.

Os teores de fósforo total excretados nas fezes não diferiu $(\mathrm{P}>0,05)$ entre as dietas que foram suplementadas com as fontes inorgânicas de fósforo. Todavia, os leitões da dieta controle (CONT- sem P suplementar) excretaram menos fósforo fecal $(\mathrm{P}<0,01)$. A excreção de fósforo total nas fezes apresentou correlação positiva $(\mathrm{r}=0,89$ e $\mathrm{P}<0,01)$ com o consumo. CUPÁK et al. (1972) e LOPES (1998), também, verificaram associação positiva entre o consumo e o teor de fósforo eliminado nas fezes. O percentual médio de fósforo excretado nas fezes, entre as dietas com fosfatos, foi de $47,2 \%$ do fósforo total consumido. Esse percentual elevou-se para $59,12 \%$ entre os leitões da dieta controle.

O fósforo urinário correlacionou-se positivamente com o $\mathrm{P}$ consumido $(\mathrm{r}=0,51 ; \mathrm{P}<0,05)$. CUPÁK et al. (1972), FERNÁNDEZ (1995a) e LOPES (1998), também, encontraram associação positiva entre o fósforo consumido e o excretado na urina, muito embora, os níveis foram divergentes dos obtidos nesta pesquisa. Todavia, os valores de fósforo metabólico urinário deste trabalho, foram relativamente semelhantes aos de VIPPERMAN JR. et al. (1974) e de BELLAVER et al. (1984), que obtiveram teores urinários diários de 26 e 26,4 mg, respectivamente, para dietas semelhantes.

Os valores de absorção aparente e real de fósforo foram semelhantes entre os tratamentos suplementados com os fosfatos $(\mathrm{P}>0,05)$, porém, os animais da dieta controle, absorveram menos fósforo do que os dos demais tratamentos $(\mathrm{P}<0,01)$. Constatou-se que o fósforo absorvido estabeleceu correlação positiva $(\mathrm{r}=0,97$ e $\mathrm{P}<0,01)$ com o $\mathrm{P}$ consumido.

CUPÁK et al. (1972) e BELLAVER et al. (1984) trabalhando com suínos em crescimento, não constataram efeito da fonte de fósforo suplementar sobre a absorção real deste mineral, o que concorda com os resultados desta pesquisa. Ainda, de acordo com estes autores, ocorreu uma menor absorção real de fósforo nas dietas sem suplementação de fósforo, quando comparado com as rações que foram suplementadas com os fosfatos.

A taxa de absorção verdadeira média do fósforo alimentar das rações contendo as fontes fosfatadas, foi de $57,02 \%$. Em dietas e metodologia semelhante, VIPPERMAN JR. et al.(1974) e BELLAVER et al. (1983) obtiveram taxas de absorção real de fósforo alimentar de 45,86 e 51,0\%, respectivamente, portanto, inferiores às deste experimento.

Resultados similares foram obtidos por 
Tabela 3 - Parâmetros relacionados ao metabolismo de fósforo de diferentes fontes, obtidos com suínos em crescimento Table 3 - Parameters connected with metabolism of the different phosphorus sources, obtained with growing swine

\begin{tabular}{|c|c|c|c|c|c|c|c|}
\hline \multirow[t]{2}{*}{$\begin{array}{l}\text { Parâmetros } \\
\text { Parameters }\end{array}$} & \multirow[t]{2}{*}{$\begin{array}{c}\text { Unidade } \\
\text { Unit }\end{array}$} & \multicolumn{6}{|c|}{$\begin{array}{c}\text { Tratamentos } \\
\text { Treatments }\end{array}$} \\
\hline & & BIC & TAP & FPM & MAP & SPT & CONT \\
\hline $\begin{array}{l}\text { Peso inicial } \\
\text { Initial weight }\end{array}$ & $\mathrm{kg}$ & $44,7^{\mathrm{A}}$ & $46,0^{\mathrm{A}}$ & $46,4^{\mathrm{A}}$ & $45,6^{\mathrm{A}}$ & $45,5^{\mathrm{A}}$ & $47,5^{\mathrm{A}}$ \\
\hline $\begin{array}{l}\text { Consumo de ração } \\
\text { Feed intake }\end{array}$ & $\mathrm{g} / \mathrm{dia}$ & $1475^{\mathrm{A}}$ & $1525^{\mathrm{A}}$ & $1450^{\mathrm{A}}$ & $1500^{\mathrm{A}}$ & $1500^{\mathrm{A}}$ & $897,5^{\mathrm{B}}$ \\
\hline $\begin{array}{l}\text { P consumido } \\
\text { P intake }\end{array}$ & $\mathrm{g} / \mathrm{dia}$ & $6,9^{\mathrm{A}}$ & $7,2^{\mathrm{A}}$ & $6,8^{\mathrm{A}}$ & $7,0^{\mathrm{A}}$ & $7,1^{\mathrm{A}}$ & $2,9^{\mathrm{B}}$ \\
\hline $\begin{array}{l}\text { P excretado fezes } \\
\text { Fecal excreted } P\end{array}$ & $\mathrm{~g} / \mathrm{dia}$ & $3,2^{\mathrm{A}}$ & $3,20^{\mathrm{A}}$ & $3,1^{\mathrm{A}}$ & $3,5^{\mathrm{A}}$ & $3,5^{\mathrm{A}}$ & $1,7^{\mathrm{B}}$ \\
\hline $\begin{array}{l}\mathrm{P} \text { absorvido aparente } \\
\text { Apparent } P \text { absortion }\end{array}$ & $\mathrm{g} / \mathrm{dia}$ & $3,7^{\mathrm{A}}$ & $3,9^{\mathrm{A}}$ & $3,7^{\mathrm{A}}$ & $3,5^{\mathrm{A}}$ & $3,6^{\mathrm{A}}$ & $1,2^{\mathrm{B}}$ \\
\hline $\begin{array}{l}\mathrm{P} \text { endógeno fecal } \\
\text { Endogenous fecal } P\end{array}$ & $\mathrm{~g} / \mathrm{dia}$ & $0,3^{\mathrm{A}}$ & $0,3^{\mathrm{A}}$ & $0,2^{\mathrm{A}}$ & $0,2^{\mathrm{A}}$ & $0,3^{\mathrm{A}}$ & $0,2^{\mathrm{A}}$ \\
\hline $\begin{array}{l}\text { P absorvido real } \\
\text { True P absortion }\end{array}$ & $\mathrm{g} / \mathrm{dia}$ & $3,9^{\mathrm{A}}$ & $4,2^{\mathrm{A}}$ & $3,9^{\mathrm{A}}$ & $3,79^{\mathrm{A}}$ & $3,9^{\mathrm{A}}$ & $1,4^{\mathrm{B}}$ \\
\hline $\begin{array}{l}\text { P urinário } \\
\text { Urinary } P\end{array}$ & $\mathrm{mg} / \mathrm{dia}$ & $25,0^{\mathrm{B}}$ & $87,0^{\mathrm{AB}}$ & $118,0^{\mathrm{A}}$ & $48,0^{\mathrm{AB}}$ & $15,0^{\mathrm{AB}}$ & $1,0^{\mathrm{B}}$ \\
\hline $\begin{array}{l}\mathrm{P} \text { retido aparente } \\
\text { Apparent Pretention }\end{array}$ & $\mathrm{g} / \mathrm{dia}$ & $3,7^{\mathrm{A}}$ & $3,8^{\mathrm{A}}$ & $3,5^{\mathrm{A}}$ & $3,5^{\mathrm{A}}$ & $3,6^{\mathrm{A}}$ & $1,2^{\mathrm{B}}$ \\
\hline $\begin{array}{l}\mathrm{P} \text { retido aparente } \\
\text { Apparent Pretention }\end{array}$ & $\mathrm{g} / \mathrm{dia}$ & $3,8^{\mathrm{A}}$ & $4,1^{\mathrm{A}}$ & $3,8^{\mathrm{A}}$ & $3,7^{\mathrm{A}}$ & $3,9^{\mathrm{A}}$ & $1,5^{\mathrm{B}}$ \\
\hline $\begin{array}{l}\text { Taxa abs. verdadeira } \\
\text { True absorption rate }\end{array}$ & $\%$ & $58,0^{\mathrm{A}}$ & $59,3^{\mathrm{A}}$ & $57,1^{\mathrm{A}}$ & $55,7^{\mathrm{A}}$ & $55,6^{\mathrm{A}}$ & $49,00^{\mathrm{B}}$ \\
\hline $\begin{array}{l}\text { Disp. biológica/fonte } \\
\text { Biol.source/available }\end{array}$ & $\%$ & $71,9^{\mathrm{A}}$ & $76,6^{\mathrm{A}}$ & $69,5^{\mathrm{A}}$ & $64,9^{\mathrm{A}}$ & $66,3^{\mathrm{A}}$ & - \\
\hline $\begin{array}{l}\text { P plasma } \\
\text { Plasm } P\end{array}$ & $\mathrm{Mg} / \mathrm{dL}$ & $8,4^{\mathrm{AB}}$ & $8,8^{\mathrm{A}}$ & $8,5^{\mathrm{A}}$ & $7,4^{\mathrm{B}}$ & $8,0^{\mathrm{AB}}$ & $8,4^{\mathrm{AB}}$ \\
\hline
\end{tabular}

* Médias seguidas de letras distintas na mesma linha, diferem entre si $(P<0,01 ; P<0,05)$.

Means, within a row, folllowed by different letters, are different $(P<.01 ; P<.05)$.

1 Considerando o $\mathrm{P}$ absorvido aparente (Considering $P$ apparent absorption).

2 Considerando o $\mathrm{P}$ absorvido real (Considering $P$ real absorption).

PARTRIDGE (1981), BELLAVER et al. (1984) e LOPES (1998) que encontraram taxas de absorção verdadeira média de 59,61;59,49; e 56,9\%, respectivamente, trabalhando com leitões em crescimento e usando a metodologia com radiotraçador $\left({ }^{32} \mathrm{P}\right)$.

A dieta controle (CONT), em que todo o fósforo contido era de origem vegetal, apresentou menor taxa de absorção real de fósforo (49\%) em relação aos demais tratamentos. Isto se deve à predominância do fósforo fítico que apresenta menor disponibilidade devido à molécula de fitato encontrar-se fortemente ligada a certos cátions, o que não permite uma hidrólise mais eficiente dos grupos ortofosfatos existentes, pela ação das enzimas do trato gastrintestinal dos monogástricos. que de acordo com KETAREN et al. (1993) possui menor disponibilidade deste mineral para os suínos. Todavia, o valor obtido está de acordo com os dados de literatura, onde a disponibilidade do fósforo de dietas à base de grãos de cereais, varia entre 18 e $60 \%$, com média de $33 \%$, para suínos de 23 a $41 \mathrm{~kg}$ (GUÉGUEN et al. 1968; NOLLAND et al.1968 e PEELER, 1972).

Os valores de fósforo retido não foram afetados $(\mathrm{P}>0,05)$ pelas diferentes fontes de fósforo suplementar. A retenção de fósforo da dieta controle, foi inferior $(\mathrm{P}<0,01)$ a das dietas contendo as fontes fosfatadas.

O fósforo retido apresentou correlação altamente positiva $(\mathrm{r}=0,97$ e $\mathrm{P}<0,01)$ com o fósforo consumido. Respostas similares foram encontradas por MUDD et al. (1969), KETAREN et al. (1993) e FERNÁNDEZ (1995a) ao constatarem relação linear significativa entre o fósforo retido e o consumido por leitões em terminação.

Os valores médios de fósforo retido por suínos de $65 \mathrm{~kg}$, consumindo 3,7; 8,1 e 13,0 g P/dia, segundo FERNÁNDEZ (1995a), foram de 1,4; 3,8 e 4,6 g P/ dia, respectivamente. Estes valores foram, relativa- 
mente, semelhantes aos desta pesquisa, resguardando as diferenças dos níveis de consumo de fósforo dietético e do peso dos leitões. Por outro lado, LOPES (1998), ao trabalhar com leitões de 47,6 kg e usando mesma metodologia, encontrou menor retenção de fósforo, sendo 1,$18 ; 1,48 ; 1,98 ; 2,95$ e $3,20 \mathrm{~g} / \mathrm{dia}$, vinculados ao consumo de 2,$97 ; 3,37 ; 4,38 ; 5,24$ e 6,78 $\mathrm{g}$ P/dia, havendo efeito linear positivo do consumo sobre a retenção de fósforo.

O fósforo retido, em relação ao absorvido aparente e real, variou de 90,59 a $92,46 \%$, entre as dietas com $\mathrm{P}$ suplementar. Isto sugere que o nível de fósforo dietético não excedeu à demanda metabólica dos leitões, ou seja, o fósforo foi eliminado pela urina em pequenas quantidades, e nas fezes, a contribuição endógena foi relativamente pequena; portanto, a retenção foi próxima do máximo, como resposta da fisiologia do organismo animal. Para ENGSTRON et al. (1985) e LOPES (1998) em dietas com fornecimento de fósforo em níveis insuficientes para atender à demanda do animal, o organismo utiliza mecanismos fisiológicos visando manter o equilíbrio homeostático do mineral na corrente sangüínea, ocorrendo o aumento da enzima 1ahidroxilase renal com o aumento no nível de 1,25 $(\mathrm{OH})_{2} \mathrm{D}_{3}$ no plasma e no intestino e, como conseqüência, aumenta a absorção de fósforo no trato gastrointestinal. Simultaneamente, os níveis do hormônio da paratireóide (paratormônio - PTH) tendem a decrescer, diminuindo a excreção de fósforo pelos rins e aumentando a reabsorção de fósforo inorgânico pelos túbulos renais. Nesta situação, certamente o transporte ativo do fósforo acoplado ao $\mathrm{Na}^{+}$ou a polipeptídeos transportadores de fósforo, é caracterizado.

A menor eficiência da retenção do fósforo absorvido $(84,14 \%)$, registrada para a dieta à base de grãos de cereais (CONT), pode estar associada, em nível de sangue, a uma discriminação metabólica do elemento em razão de sua origem (GUÉGUEN et al., 1968; VITTI et al., 1989). Resultados semelhantes foram obtidos por BELLAVER et al. (1984) e LOPES (1998) que verificaram menor eficiência de retenção de fósforo das dietas à base de milho e farelo de soja, sem a suplementação de fósforo; contudo, os valores obtidos por estes pesquisadores foram inferiores aos registrados nesta pesquisa. Por outro lado, CUPÁK et al. (1972) e BELLAVER et al. (1983) não constataram diferença no balanço de fósforo entre as dietas com e sem P suplementar, tendo inclusive observado percentuais mais elevados de retenção de fósforo, com variação de 95,48 a 98,9\%.
A disponibilidade biológica (DB) do fósforo suplementar (DBPS) variou de 64,92 a $76,67 \%$ e não diferiu $(\mathrm{P}>0,05)$ entre as fontes fosfatadas. Estes resultados foram superiores aos de BELLAVER et al (1984); PARTRIDGE ( 1981) e FARIA ( 1995 ). $\mathrm{O}$ fósforo endógeno fecal e a DB do fósforo da ração com fosfato bicálcico, não diferiram dos valores encontrados para as rações com os fosfatos naturais de rocha, o que está de acordo com BROCE (1986).

O teor de fósforo no plasma não mostrou associação significativa com o consumo deste elemento. LOPES (1998), em pesquisa semelhante, não verificou relação entre esta variável e a ingestão de fósforo, certamente, em função do tempo curto dos experimentos. Por outro lado, KOCH e MAHAN (1986) e ENGSTRON et al. (1985) encontraram efeito linear significativo na concentração de fósforo inorgânico no soro sangüíneo de suínos em terminação com os níveis de fósforo dietético e verificaram que a hipofosfatemia se instalou progressivamente nos leitões alimentados com as dietas deficientes em fósforo, atingindo média mínima de fósforo no plasma, em torno de 34 a 41 dias de restrição do mineral na dieta, como conseqüência da depleção das reservas lábeis do elemento nos tecidos de reserva de fósforo no organismo animal.

\section{Conclusões}

Como o P excretado nas fezes, o P endógeno fecal, a absorção e a rentenção de $P$ não foram afetados pelas diferentes fontes utilizadas, concluiuse que a disponibilidade biológica do $\mathrm{P}$ dos fosfatos de rocha foi similar à do $\mathrm{P}$ do fosfato bicálcico.

\section{Referências Bibliográficas}

ASSOCIATION OF OFFICIAL ANALYTICAL CHEMISTS AOAC. 1980. Official methods of analysis. 13.ed. Washington, D.C. $1018 \mathrm{p}$.

BELLAVER, C., GOMES, P.C., SANTOS, D.L. 1983. Absorção e disponibilidade de fósforo para suínos, baseada na diluição de radiofósforo ( ${ }^{32} \mathrm{P}$ ). Pesq. Agropec. Bras., 18(9):1053-1057.

BELlAVER, C., GOMES, P.C., FIALHO, E.T. et al. 1984. Absorção e disponibilidade do fósforo de fosfatos naturais em rações para suínos. Pesq. Agropec. Bras., 19(12):1513-1518.

BROCE, C.S. Comparação entre diferentes fontes de fósforo em rações para suínos nas fases de crescimento e terminação. Santa Maria: UFSM, 1986. Dissertação (Mestrado em Zootecnia) - Universidade Federal de Santa Maria, 1986.

COMAR, C.L., MONROE, R.A., VISEK, W.J. et al. 1953. Comparison of two isotope methods for determination of endogenous fecal calcium. J. Nutr., 50: 459-467.

CRENSHAW, T.D., PEO JÚNIOR, E.R., LEWIS, A.J. et al. 1981. Influence of age, sex and calcium and phosphorus levels 
on the mechanical properties of various bone in swine. J. Anim. Sci., 52(6):1319-1329.

CUPÁK, M., PROCHAZKA, Z., JAMBOR, V. 1972. Utilization of phosphorus compounds in pigs after endogenous phosphorus determination by of ${ }^{32} \mathrm{P}$. Acta Veter. Brno, 41:257-262.

DeLUCA, H.F. 1979. The vitamin D system in the regulation of calcium and phosphorus metabolism. Nutr. Ver., 37:161-163.

ENGSTRON, G.W., HORST, R.L., REINHARDT, T.A. et al. 1985. Effect of dietary phosphorus levels on porcine renal 25-hydroxyvitamin D, 1a and 24-R hydroxylase activities and plasma 1,25-dihydroxyvitamin $\mathrm{D}_{3}$ concentration. J. Anim. Sci., 60:1005-1011.

FARIA, S.R. Determinação da disponibilidade de fósforo $e$ avaliação do desempenho de suínos em cinco fontes fosfatadas. Belo Horizonte: UFMG, 1995. 85p. Dissertação (Mestrado) - Universidade Federal de Minas Gerais, 1995.

FERNÁNDEZ, J.A. 1995. Calcium and phosphorus metabolism in growing pigs. I. Absorption and balance studies. Livest. Prod. Sci., 41(3):233-242.

FIGUEIRÊDO, A.V. 1998. Disponibilidade biológica do fósforo de cinco fosfatos, determinada em suinos em crescimento, através da técnica de diluição isotópica. Piracicaba: CENA, 1998. 103p. Tese ( Doutorado em Ciência Animal) - Centro de Energia Nuclear na Agricultura, Universidade de São Paulo, 1998.

FISKE, C.H., SUBBARROW, Y. 1925. The colorimetric determination of phosphorus. J. Biol. Chem., 66(2):375-400.

GUÉGUEN, L., BENSANÇON, P., RERART, A. 1968. Utilization disgestive, cinétique de l'absorption et efficacité de la rétention du phosphore phytique chez le porc. Annales de Biol. Anim. Bioc. Biop., 8(2):273-280.

INTERNATIONAL ATOMIC ENERGY AGENCY - IAEA. 1979. Laboratory training manual on the use of nuclear technique in animal research. Viena. 299p. (Technical Report Series, 193).

KETAREN, P.P., BATTERHAM, E.S., DETTMANN, E.B. et al.1993. Phosphorus studies in pig. 3. Effect of phytase supplementation on the digestibilitymeal for grower pigs. Brit. J. Nutr., 70:289-311.

KOCH, M.E., MAHAN, D.C. 1986. Biological characteristics for assessing low phosphorus intake in finishing swine. J. Anim. Sci., 62:163-172.

LOFGREEN, G.P. 1960. The availability of the phosphorus in dicalcium phosphate, bone meal, soft phosphate and calcium phytates for mature wethers. J. Nutr., 70(1):58-62.

LOPES, J.B. Avaliação da absorção real e das perdas endógenas de fósforo em suínos pela técnica de diluição isotópica. Piracicaba, SP: USP, 1998. 87p. Tese (Doutorado em Ciência Animal) - Centro de Energia Nuclear na Agricultura (CENA)/ Universidade de São Paulo, 1998.
MAXSON, P.F., MAHAN, D.C. 1983. Dietary calcium and phosphorus levels for growing swine from 18 to 57 kilograms body wigth. J. Anim. Sci., 56(5):1124-1134.

MUDD, A.J., SMITH, W.C., ARMSGTRONG, D.G. 1969. The influence of dietary concentration of calcium and phosphorus on their retention in the body of the growing pig. J. Anim. Sci., 73:189-198.

NASCIMENTO FILHO, V.F., LOBÃO, A.O. 1977. Detecção de p-32 em amostras de origem animal e vegetal por efeito Cerenkov, cintilação líquida e detector GM. Piracicaba: CENA. 25p. (Boletim Científico, 48).

NATIONAL RESEARCH COUNCIL - NRC. 1988. Nutrient requirements of swine. 9.ed. Washington, D.C. 93p.

NOLAND, P.R., FUNDERBURG, M., JOHNSON, Z. 1968. Phosphorus availability in practical diet for swine. J. Anim. Sci., 27(4): 115.

PARTRIDGE, I.G. 1980. Mineral nutrition of the pig. Proc. Nutr. Scoc., 39(2): 185-192.

PARTRIDGE, I.G. 1981. A comparison of defluorinated rock phosphate and dicalcium phosphate, in diets containing either skim milk powder or soybean meal as the main protein supplement for early weaned pigs. Anim. Prod., 3(1): 67-73.

PEELER, H.T. 1972. Biological availability of nutrients in feeds: availability of major mineral ions. J. Anim. Sci., 35(3):695712.

ROSTAGNO, H.S., SILVA, D.J., COSTA, P.M.A. et al. 1987. Composição de alimentos e exigências nutricionais de aves e suínos: tabelas brasileiras. Viçosa: UFV. 59p.

SARRUGE, J.R., HAAG. H.P. 1974. Análises químicas em plantas. Piracicaba: ESALQ/USP. 56p.

TONROY, B., PLUMLEE, M.P., CONRAD, J.H. et al. 1973. Apparent digestibility of the phosphorus in sorghum grain and soybean meal for growing swine. J. Anim. Sci., 36(4):669-673.

VIPPERMAN JR., P.E., PEO JR., E.R., CUNNINGHAM, P.J. 1974. Effect of dietary calcium and phosphorus level upon calcium, phosphorus and nitrogen balance in swine. J. Anim. Sci., 38(4):758-765.

VITTI, D.M.S.S. Avaliação da disponibilidade biológica de fósforo dos fosfatos bicálcico, Patos de Minas, Tapira e finos de Tapira para ovinos pela técnica de diluição isotópica. São Paulo: IPEN, 1989. 87p. Tese (Doutorado) - Instituto de Pesquisas Energéticas e Nucleares, 1989. 